\title{
Understanding Event Related Potentials during Reading using Pre-Compiled Abstractive Decoders
}

\author{
Shaorong Yan \\ Department of Brain and Cognitive Sciences \\ University of Rochester \\ Rochester, NY 14627, USA \\ syan13@ur.rochester.edu \\ Aaron S. White \\ Deparment of Linguistics \\ University of Rochester \\ Rochester, NY 14627, USA \\ aaron.whitedrochester.edu
}

\begin{abstract}
ERPs have been an important tool in studying the time course of information integration in language processing. While most of the ERP studies have used language materials designed by the researchers, in recent years, there have been a growing interest in applying ERPs in the processing of natural stimuli. This calls for more exploratory studies and brings challenges to traditional analytical methods. In the current paper, we develop a new analytical tool to link language predictors to ERP signals. Specifically, we use the decoder component of pre-trained convolutional autoencoders (CAEs) to decode ERPs from predictors of language models. We validate the model by showing that it can replicate findings using more conventional analytical methods. Use this model, we examine the ERP correlates of static (GloVe) and contextualized (ELMo) word embeddings. We show that word embeddings explain both early ERP signals that are more related to form-based processing (e.g., N1/P1, N250) and ERP components that are more related to meaning processing (e.g., N400). Lastly, we discuss the potential application of this framework for future studies.
\end{abstract}

\section{Introduction}

Understanding the mechanisms by which comprehenders incrementally process linguistic input in real time has been a key endeavor of cognitive scientists and psycholinguists. Due to its fine temporal resolution, event-related potentials (ERPs) have been an effective tool in probing the rapid, online cognitive processes underlying language comprehension. Traditionally, most language processing studies 
using ERPs have focused on how different ERP components-systematic shifts in ERP amplitudes that have a specific temporal and spatial profile (for an overview, see Luck, 2014) — are affected by various properties of linguistic inputs (see Van Petten and Luka, 2012; Kuperberg, 2016, for reviews). For example, the N400 is a negative-going ERP component that peaks at centro-parietal electrodes around 400ms after stimulus onset (Kutas and Hillyard, 1980) and is sensitive to how expected the meaning of the language input is (for review, see Kutas and Federmeier, 2011). A major aim of such studies is to determine what types of linguistic information are associated with different ERP components-e.g. the N1/P2 (Sereno et al., 1998; Dambacher et al., 2006), N250 (Grainger et al., 2006), N400 (Kutas and Hillyard, 1980; Hagoort et al., 2004; Lau et al., 2008), and P600 (Osterhout and Holcomb, 1992; Kuperberg et al., 2003; Kim and Osterhout, 2005) - with the aim of understanding when different types of information is accessed over the course of linguistic processing.

When analyzing the effect of linguistic predictors on different ERP components, researchers typically aggregate ERP amplitudes across time samples and electrodes based on knowledge about the temporal and spatial distribution of known components (e.g., Frank et al., 2015; Dambacher et al., 2006). While this approach has been fruitful, researchers have also long been aware of the potential drawbacks to this component-based approach. First, aggregating across time points sacrifices temporal resolution: a predictor's effects can be too transient to detect when averaging ERP amplitudes within a time window (see Hauk et al., 2006, Yan and Jaeger, 2019, for discussion). This is exacerbated if the time course of the effect varies in its time course across subjects and items. Second, different predictors can affect ERPs in the same time window of an established component but have slightly different temporal (Frank and Willems, 2017) or spatial (DeLong et al., $2005)$ profiles. This means that the operative definition of a component-i.e. how one aggregates across time samples and electrodes—can sometimes strongly affect the interpretation of results.

The drawbacks of such component-based analyses are especially pronounced in exploratory studies that focus on naturalistic inputs-e.g. sentences from novels (Frank et al., 2015; Frank and Willems, 2017) or excerpts from audiobooks (Broderick et al., 2018). In such studies, one tends to have fewer a priori expectations as to when and where some effect will reveal itself, compared to confirmatory studies that explicitly manipulate some set of linguistic properties (e.g., DeLong et al., 2005: Federmeier and Kutas, 1999; Kim and Osterhout, 2005, Dambacher et al., 2006). This challenge is pressing in light of the field's increasing adoption of naturalistic stimuli as a means to improve the ecological validity of ERP research (for discussion, see Yan and Jaeger. 2019). This challenge arises, at least in part, because when using natural stimuli, the properties of the language input often need to be extracted and modeled post hoc, massively increasing the degrees of freedom invoked in the analysis.

There are at least two possible approaches to this challenge. One possibility is to plot the data and use visual inspection to select an analysis plan that is 
specifically tailored to the dataset. This approach is problematic because it introduces uncontrollable researcher degrees of freedom (Gelman and Loken, 2014) and thereby makes results less comparable across studies. Therefore the more commonly adopted approach is to run separate models for each time point (or even each electrode) to look for the emergence of effects. This second approach necessitates complex statistical tests to monitor for inflated Type I error (e.g., nonparametric permutation tests Blair and Karniski, 1993) and to control for autocorrelation across time points (Smith and Kutas, 2015; Piai et al., 2015; Yan and Jaeger, 2019); and because it is computationally-intensive, researchers sometimes only focus on a limited number of electrodes in their analyses (see, e.g., Laszlo and Federmeier, 2014, Frank and Willems, 2017; Yan and Jaeger, 2019). While these technical issues are surmountable using sophisticated statistical models and more computing power, this approach to analysis is faced with an inherent conceptual problem: it targets the wrong level of abstraction. Rather than focusing on the holistic structure of the signal viewed as the product of many different underlying processes, this approach takes samples and electrodes as the fundamental units of analysis.

We propose an alternative approach to the analysis of ERPs that addresses these problems head on. The main idea behind our approach is to automatically construct abstractions that capture the holistic structural properties of ERP signals using tools originally developed for a similar purpose in the domain of computer vision: convolutional autoencoders (CAEs, Masci et al., 2011). Inspired by multimodal machine learning tasks, like image captioning (see Hossain et al., 2019. for a review) and visual question answering (Antol et al., 2015), we use the mapping from abstractions to raw ERP signals learned by our CAE as a function with which any quantitative model of language processing can be composed by automatically inferring a mapping from the language processing model's predictions into the space of abstractions built using the CAE. Because this approach is agnostic about the exact nature of the language processing model, it enables explicitly quantitative comparison of different language models within a unified framework. Further, because our CAEs are built across all time points and electrodes making up a signal, we avoid the need to select particular spatial or temporal regions for analysis, thereby reducing researchers' degrees of freedom.

To demonstrate the efficacy of our approach, we build on recent work on the prediction of ERP components using probabilistic (Frank et al., 2015) or neural network-based (Ettinger et al., 2016; Frank and Willems, 2017; Hale et al., 2018) language models. We begin in Section 2 by discussing how we train CAEs to construct abstractions with varying levels of compression as well as how we use these trained CAEs to decode ERPs from language model predictors. In Section 3, we examine the nature of the abstractions learned by our CAEs as well as how different kinds of language model predictors affect the decoding of ERPs when paired with different autoencoders, including language predictors whose effects have previously been examined on the same dataset (lexical frequency, lexical surprisal, and semantic distance Frank and Willems, 2017; Yan and Jaeger, 2019). We exam- 


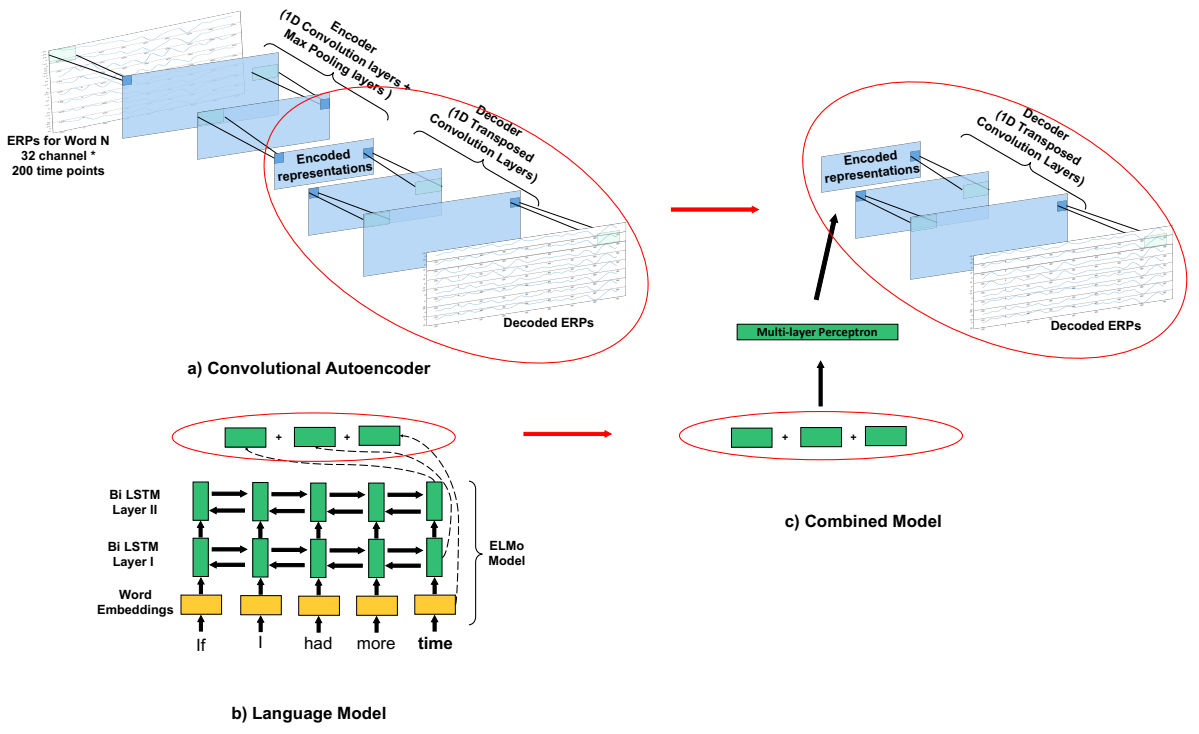

Figure 1: An instance of our framework using a bidirectional language model (ELMo) as the text encoder. a) Structure of the convolutional autoencoder. b) Stucture of the language model (ELMo). c) Structure of the combined model that decode ERPs from language models using a pre-trained decoder.

ine how well different language model predictors capture various spatiotemporal properties of the signal, relating our findings back to prior confirmatory work. In Section 4, we conclude with some limitations of and future directions for the current approach.

\section{Pre-compiling abstractive decoders}

In this section, we describe how we train our convolutional autoencoders (CAEs) to extract latent structures in ERPs. We begin with background on the mathematics of CAEs $(\$ 2.1$, and then discuss the range of autoencoder architectures we consider (\$2.2) as well as the particular EEG data we fit our models to (\$2.3). We then turn to the method we use to train $(\$ 2.4)$ and evaluat $(\$ 2.5)$ these models and results $(\$ 2.6)$.

\subsection{Convolutional autoencoders}

Autoencoders are a family of unsupervised neural network models that search for a compressed representation of some data from which the original data can be reconstructed with little loss of fidelity (for introduction and review, see Bengio et al., 2013, Hinton and Zemel, 1994). They are composed of three fundamental components: (i) an encoder that maps the original data into the compressed representations; (ii) a decoder that maps the compressed representation to a reconstruction of the original data; and (iii) a measure of reconstruction infidelity or loss. The loss 
function can be roughly thought of as the distance between the reconstructed data and the original data, with common loss functions being the mean absolute error or mean squared error. The model can then be trained through back propagation to find the latent representation that minimizes this difference.

In the current paper, we use convolutional autoencoders (see Figure 1 1 , for illustration) to model ERPs. This is a type of autoencoder where both the encoder and decoder components are convolutional neural networks (CNNs, for a recent review, see Rawat and Wang, 2017). CNNs have been widely used to abstract information from complex, multi-dimensional data like images (Krizhevsky et al., 2012), videos (Karpathy et al., 2014), and text (Kim, 2014). Since ERPs are also a high dimensional data that unfold in both space and time, we explore whether one can also use CNNs to encode and decode ERPs and reveal underlying structures.

CNNs are typically composed of a series of convolutional layers interleaved with pooling layers. A convolutional layer (illustrated in Figure 2) consists of a set of filters, where each filter takes in a weighted sum of the outputs $\left(x_{i}\right)$ from all the nodes $(i)$ within its "receptive field" $(F)$ and pass it through a nonlinear activation function $\sigma$-commonly a hyperbolic tangent or rectifier-to generate one single output $\left(o_{j}\right.$, see Equation 11).

$$
o_{j}=\sigma\left(\sum_{i}^{F} w_{i j} x_{i}+b_{j}\right)
$$

Each filter is applied to the entire input layer with the same set of weights $\left(w_{i j}\right)$, moving along each dimension of the input. Each filter's receptive fields, when applied at different spatial locations, may or may not overlap with each other. This is controlled by the stride size parameter (see Figure 2). Such architecture allows the model to reveal patterns that are spatially and temporally dependent ${ }^{1}$ The main use of these parameters in our experiments is to capture different frequency bands within the EEG signal.

Pooling layers have the same architecture as convolutional layers; but instead of point-wise applying an activation function, they apply some aggregation function to their receptive field (see Equation 2). A common choice is max-pooling, where the maximal value within receptive field becomes the output.

$$
o_{j}=\sigma_{i \in F}\left(x_{i}\right)
$$

Using these two components, the encoder gradually shrinks the size of the input and creates a latent representation of the input data. These abstractions can be thought of as compressing complex patterns into a single number. (We demonstrate the kinds of complex patterns such networks find in EEG below.)

The decoder component, in contrast, is composed of a series of transposed convolutional layers or deconvolutions. Transposed convolutional layers consist of

\footnotetext{
${ }^{1}$ The ability to capture temporal dependence comes from the fact that time is represented as one dimension of the matrix with which we represent EEG recordings. See $\$ 2.2$ for details.
} 


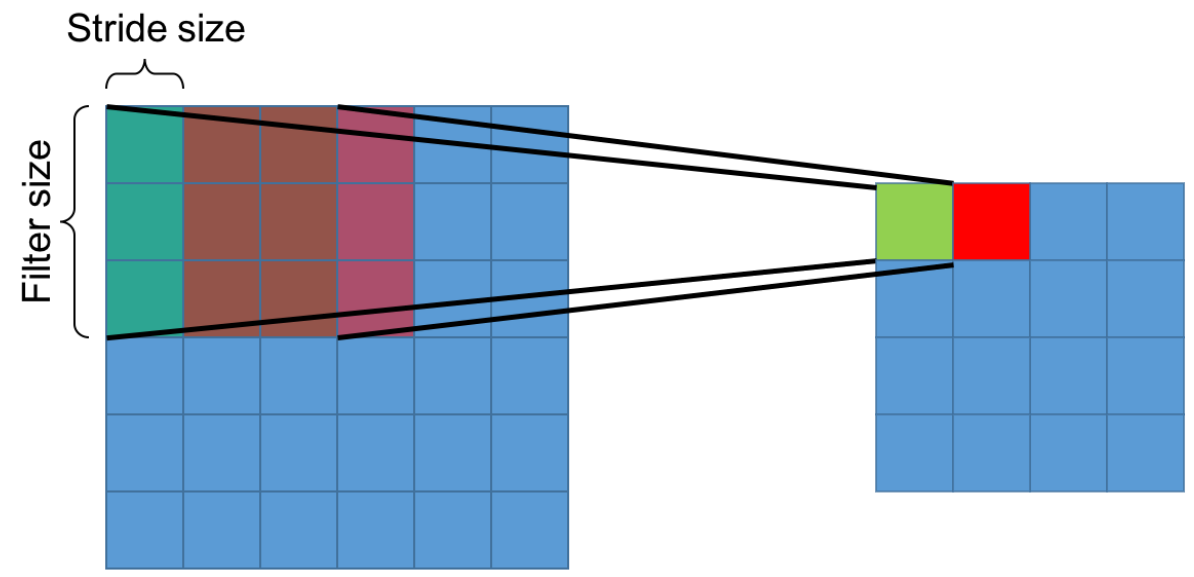

Figure 2: Illustration of a convolutional layer, with a filter size of $3 * 3$ and a stride size of 1 .

filters of the opposite shape as those in the convolutional layers. The filters take input from one node and generate multiple outputs within its output receptive field. Layer by layer, the decoder reprojects the latent representation back to the same size as the input data. One can then calculate the distance the reconstructed data and actual input and use it as the loss function to train the model.

In the following parts, we describe how we design the structures of our CAEs and how we train and evaluate them in the specific study. Lastly, we present the performance of the autoencoders on actual EEG recordings during reading.

\subsection{Model structure}

Our CAEs consist of two components: (a) a convolutional encoder that finds a way to best compress the ERP signals; and (b) a convolutional decoder with an isomorphic architecture that reconstructs the ERP data from the compressed representation. ERPs are organized into a $2 \mathrm{D}$ matrix (channel $\times$ time points). We first pass the ERPs through a linear transformation to allow for recombination of different electrodes. This linear layer is then linked to the encoder side of the autoencoder. The encoder consists of a series of convolutional layers interleaved with maxpooling layers. Correspondingly, for the decoder part, we use a homomorphic series of 1D transposed convolutional layers to reconstruct the ERP data. The last layer of the decoder is also connected to a linear layer that allow for recombination of the latent electrodes before getting compared to the amplitudes of the actual ERPs.

We perform an architecture search over autoencoders with different levels of compression and examine which autoencoder can best compress the data for reconstruction. The selection of model structures is informed by the major EEG frequency bands. For example, the model with the highest level of compression 
has 5 nodes along the time domain. Given the length of the epoch $(800 \mathrm{~ms})$, this would best preserve information that are around $6 \mathrm{~Hz}$, thus within the theta band frequency range (hence theta models). In similar fashion, we also construct models that approximately correspond to alpha, beta, and gamma band frequency at the most compressed layer. Henceforth, we use the approximately encoded frequency band as a shorthand to refer to each of our autoencoder models.

At each layer, we also shrink the number of latent channels in order to (i) discover higher-level patterns of activation across time; and (ii) combine redundancy across the channels into a single parameter. At each step the number of channels is shrunk by a factor of 2 . To examine how many latent channels are needed to represent the internal state, we also vary the width of the model. The wide models have twice as many latent channels as the narrow model after transformation at each layer. Table 1 lists the number of time nodes and the number of latent channels after each steps of compression.

\begin{tabular}{ccc}
\hline Model & $\begin{array}{c}\text { NO. of } \\
\text { time nodes }\end{array}$ & $\begin{array}{c}\text { NO. of } \\
\text { latent channels } \\
\text { (Wide/Narrow) }\end{array}$ \\
\hline Theta & 100 & $32 / 32$ \\
& 34 & $32 / 16$ \\
& 17 & $16 / 8$ \\
& 9 & $8 / 4$ \\
& 5 & $4 / 2$ \\
\hline Alpha & 100 & $32 / 32$ \\
& 34 & $32 / 16$ \\
& 17 & $16 / 8$ \\
& 9 & $8 / 4$ \\
\hline Beta & 100 & $32 / 32$ \\
& 34 & $32 / 16$ \\
& 17 & $16 / 8$ \\
\hline Gamma & 100 & $32 / 32$ \\
& 34 & $32 / 16$ \\
\hline
\end{tabular}

Table 1: Number of latent time nodes and latent channels after each step of compression for each autoencoder tested in the architecture search.

\subsection{EEG data}

We use the EEG recordings collected by Frank et al. (2015), shared by the first author of that study. In Frank et al's 2015 study, 24 subjects read sentences drawn from three novels. Sentences were presented word-by-word using a rapid serial visual presentation paradigm, with varying stimulus onset asynchrony (SOA) as a function of word length. We use the ERPs of each word epoched from -100 to $700 \mathrm{~ms}$ time-locked to word onset from all the 32 recorded scalp channels. The 
dataset has a sampling rate of $250 \mathrm{~Hz}$, thus we have data from 200 time points for each word. Since some effects we are interested in are contextual (e.g., semantic distance) that require at least one preceding word to compute, we do not include the first word of the sentence for the training of our models. This left ERPs for 1,618 word tokens per subject (638 word types). After excluding trials containing artifacts (as annotated in the EEG data by Frank et al.), a total of 37,112 training instances remain.

\subsection{Model training}

All models are trained with mean squared error loss between the ERPs reconstructed by the autoencoder and the actual ERPs. Model weights are updated using mini-batch gradient descent (batch size $=128$ ) with the Adam optimizer (learning rate $=0.001$ and default settings for beta1, beta2, and epsilon) implemented in pytorch (Paszke et al., 2017). Each model is updated for 100 epochs. Post-hoc check show that model performance on the development set has stopped improving at the end of the training.

We perform a hyper-parameter tuning over three levels of weight decays $\left(10^{-1}\right.$, $10^{-3}, 10^{-5}$ ) using 5 -fold cross-validation. The folds are divided either across sentences or across subjects: We first train the model with ERPs on word from 4/5 of all the sentences/subjects (training set) and then tested it on the rest 1/5 (development set). To further control for overfitting, we adopted early stopping-i.e. we select the models that give the best performance on the development set during training.

\subsection{Model evaluation}

We evaluate the performance of each model by comparing the $R^{2}$ between reconstructed ERPs and actual ERPs on the development set. Specifically, we compared the models to a baseline model that only predicts the mean amplitude across time points and electrodes of each training set. The mean squared error of this baseline model is thus the variance of the development set (VAR). The $R^{2}$ of each autoencoder is then calculated using the following formula:

$$
R^{2}=1-\frac{\text { MSE }_{\mathrm{CAE}}}{\mathrm{VAR}}
$$

\subsection{Results}

We first summarize the overall performance of each autoencoder measured by $R^{2}$ (\$2.6.1), then provide some more direct insight into what information the autoencoders capture by visualizing both the decoded ERPs and the encoded states $(\$ 2.6 .2$. 


\subsubsection{Overall performance}

Table 2 shows the overall performance of each autoencoder that we examine. We evaluate the model performance using $R^{2}$. All presented results are the mean $R^{2}$ on the development set in cross-validation. Unsurprisingly, the models with lower compression rate overall has better performance than the models with lower compression rate (see also Figure 3), presumably because the less compressed models have more nodes along the time axis and preserved more fine-grained temporal information. Besides, overall the wide models have better performance than the narrow models. This difference is more pronounced for models that have a smaller encoded state (higher compression rate, e.g., the theta model) but less so for models with lower compression rate like the gamma model. Lastly, the autoencoders perform equally well when doing cross-validation across sentences or across subjects.

\begin{tabular}{cccccc}
\hline \multirow{2}{*}{ Model } & \multicolumn{2}{c}{ Wide } & \multicolumn{2}{c}{ Narrow } \\
\cline { 3 - 6 } & & $R^{2}$ & CI & $R^{2}$ & CI \\
\hline \multirow{2}{*}{ Theta } & by-Sentence & 43.6 & {$[37.6,49.5]$} & 29.5 & {$[25.2,33.2]$} \\
& by-Subject & 43.3 & {$[39.5,47.4]$} & 30.4 & {$[25.4,34.8]$} \\
\hline \multirow{2}{*}{ Alpha } & by-Sentence & 62.1 & {$[56.6,67.1]$} & 52.7 & {$[48.4,56.9]$} \\
& by-Subject & 60.6 & {$[57.2,64.1]$} & 49.7 & {$[45.8,54.8]$} \\
\hline \multirow{2}{*}{ Beta } & by-Sentence & 75.2 & {$[73.6,76.8]$} & 67.8 & {$[65.5,70.2]$} \\
& by-Subject & 74.8 & {$[73.1,76.6]$} & 67.6 & {$[65.2,69.9]$} \\
\hline \multirow{2}{*}{ Gamma } & by-Sentence & 85.2 & {$[83.6,86.7]$} & 81.8 & {$[79.9,83.7]$} \\
& by-Subject & 84.5 & {$[82.9,85.7]$} & 81.5 & {$[79.7,83.2]$} \\
\hline
\end{tabular}

Table 2: Proportion of total variance $\left(R^{2}\right)$ explained by each autoencoder $(\times 100)$ and bootstrapped confidence interval across folds.

To illustrate how well each autoencoder reconstructs ERPs, we plot the decoded ERPs and the original ERPs of an example trial in Figure 4 on three mid-line electrodes at frontal, central and parietal parts of the scalp. As shown in the Figure, all autoencoders reconstruct the overall trend of the ERPs and the performance is also very comparable across the different sites both when cross-validated across sentences and across subjects. Suggesting that the auto-encoders have good generalizability across both sentences and subjects. The theta model that has the highest compression rate only preserve lower frequency information and does not keep much of the alpha band activity that is ample in the signals. This likely explains why the performance of theta models is not as good as the other models.

\subsubsection{Encoded states}

To further unpack what patterns are extracted by the autoencoders, we activate the encoded layers of the decoder part of the autoencoder and examine what is the activation pattern at the final layer of the decoder. The activation is then aggregated 


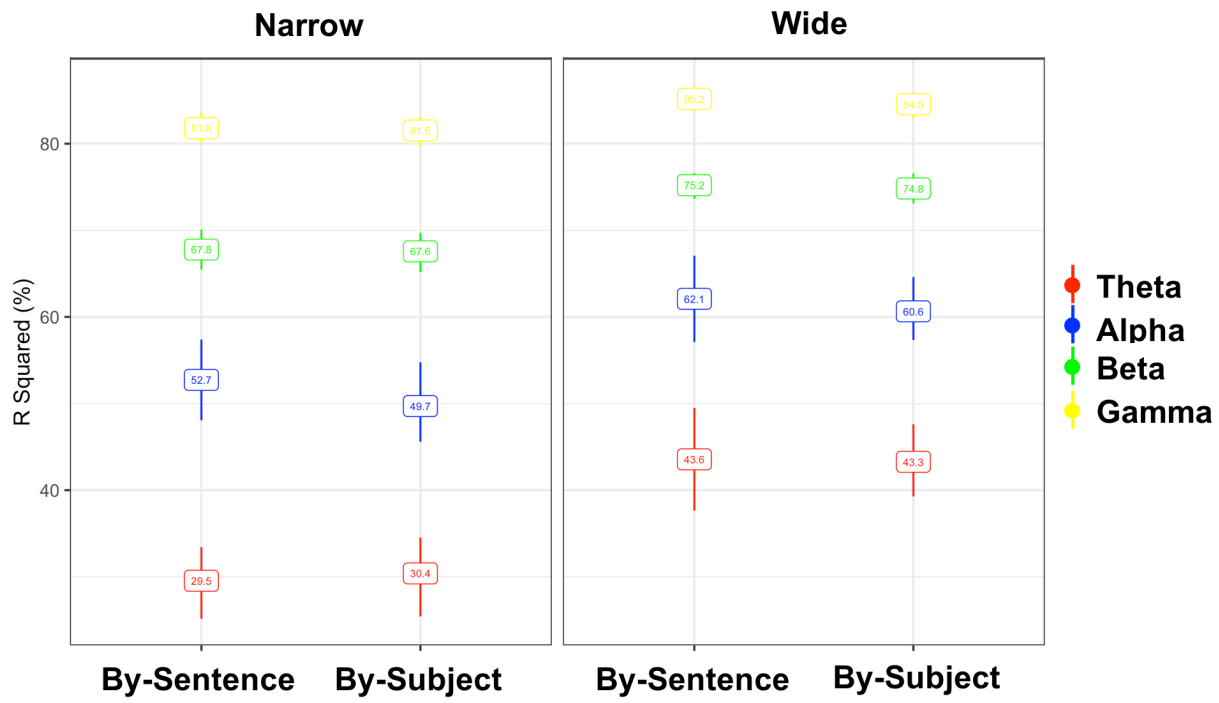

Figure 3: Proportion of total variance $\left(R^{2}\right)$ explained by each autoencoder $(\times 100)$ with bootstrapped confidence interval across folds.

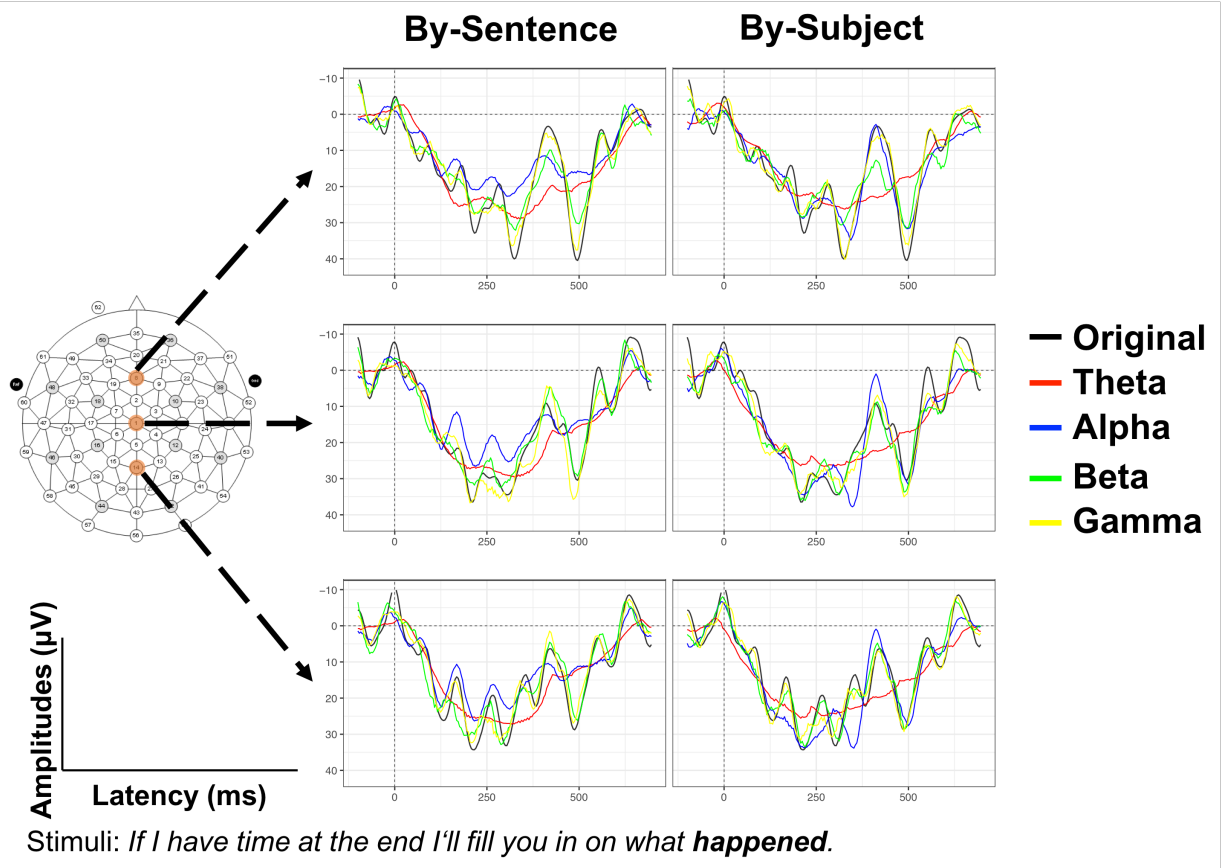

Figure 4: ERPs decoded from each autoencoder from three mid-line electrodes $(\mathrm{Fz}, \mathrm{Cz}, \mathrm{Pz})$ of one trial from one subject. Colored lines represent decoded ERPs from each autoencoder. Black lines represent original ERPs. 

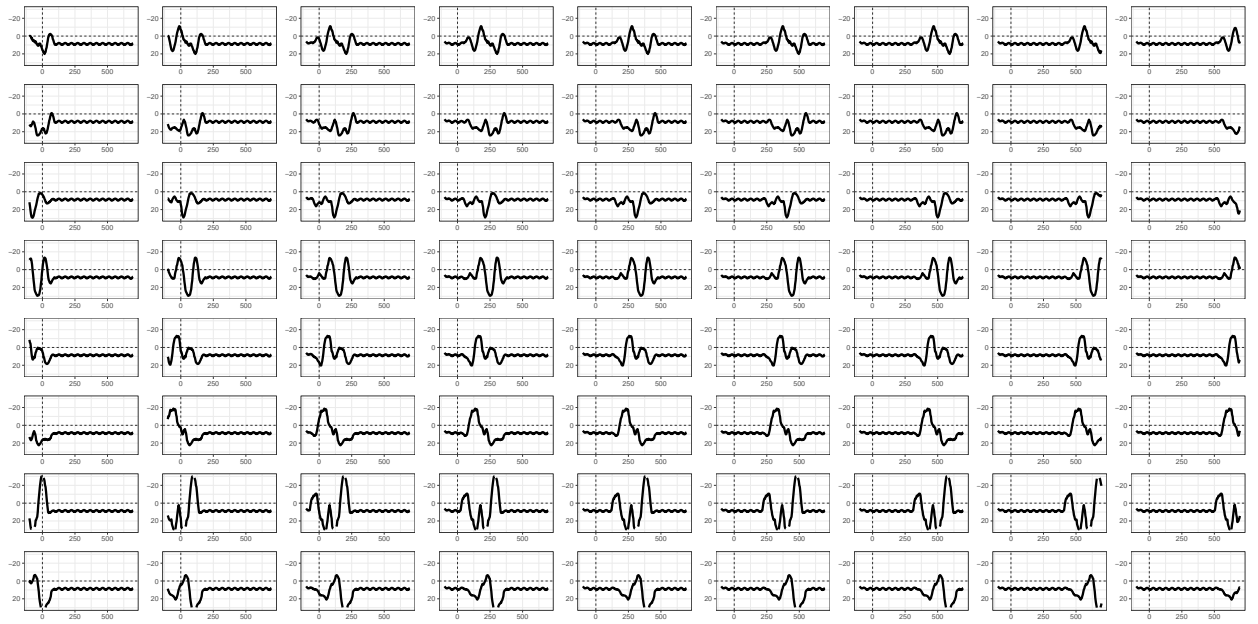

Figure 5: Average activations in the output layer of the alpha encoder resulting from individually activating each node in the encoded representation. Rows and columns correspond to the location of the node in the encoded representation.

across electrodes to illustrate the activation over time. Figure 5 showcases the activation pattern of one of the alpha models we trained. Each row represents a different latent channel while each column corresponds to a latent time step. Each vignette corresponds to the activation pattern in the last layer when activating the node at each latent channel and latent time step combination. Because we use 1D convolution filters, each latent channel produces a pattern that is shifted in time. With this set up, the shift in time is approximately $100 \mathrm{~Hz}$, comparable to the alpha band frequency. While all channels have similar temporal profile, they each produce slightly different activation pattern that can be consequently combined to form more complex patterns to resemble ERPs.

\subsection{Discussion}

We show that CAEs can reconstruct ERPs well from a compressed representation. This is a sanity check to show that the specific structures we design for modeling ERPs can indeed reconstruct the underlying signals reasonably well. Even the models with just a small number of nodes in the compressed state are able to reconstruct a large proportion of the signals in the ERPs (see Figure 3). For example, the most compressed layer of our (wide) alpha models only have 8 latent channels and 9 nodes along the time domain, but it can reconstruct over $60 \%$ of the variance in the signals that have 32 channels and 200 nodes along the time domain.

Further, the performance of the CAEs does not differ much when cross-validated across subjects or across items. This suggests that the latent structures unveiled by the CAEs can be well generalized both across subjects or items. As shown in Figure 5, the CAEs seem to rely on a set of simple filters that have slightly different 
shapes that are distributed across time and space.

While the more compressed models already capture the bulk of the overall variance in the dataset, the models with more nodes along the time dimension overall have better performance-as do models that have more latent channels. This is unsurprising because models have more nodes along the time axis can encode higher frequency information in the data, as shown in Figure 4, and model with more channels can encode more filter types. However, it is unclear whether these signals are related to language processing and thus perform better in decoding ERPs from language predictors. We empirically examine this question in the following section.

\section{Combined models}

In this section, we will take the different autoencoders trained in the previous section and link them with different language predictors. The basic recipe is to detach the decoder component of the CAEs, freeze their weights, and use them to reconstruct ERPs from language predictors. Specifically, we pass each language predictor through a neural network to project them onto the same dimensionality and size as the latent representation of each autoencoder, from which we then use the corresponding decoder to decode ERPs. An example model structure using ELMo embeddings (Peters et al., 2018) is illustrated in Figure 1.

Instead of only using the CAEs that have the best reconstruction performance, we examined the performance of all CAE structures when they are combined with language predictors. The rationale behind this is that, while the CAEs with less compression can better reconstruct ERPs, when combined with the language models, the performance might not necessarily be better.

There are two reasons why this might be the case. First, a higher compression rate can potentially force the model to focus on the most salient features of the ERP signals that are the most systematic and task relevant. Second, when the compression rate is lower, the compressed representation from the autoencoder will have more nodes. Consequently, when combined with language predictors, especially the word embeddings that have as many as over a thousand nodes, lower compression rates will greatly inflate the number of parameters that need to be trained. This provides challenges for the optimization process and also increases the risk of overfitting. The ultimate test thus is which decoder, when combined with the language model, has the best performance in revealing the effects of each predictor on ERP data. Therefore, rather than only choosing the autoencoder with the best performance, we use the best performing autoencoder at each compression rate for the language predictor models. 


\subsection{Model structure}

All of the combined models have two components: (i) the decoder component from our pre-trained CAEs that decode ERPs time-locked to word onsets from a latent representation; and (ii) a language model from which features can be extracted for each word-e.g. the surprisal of that word given previous words or its contextual word embeddings.

\subsection{EEG data}

The same EEG data set used to train the CAEs are used to train and evaluate the combined models. We note that we train the autoencoders and the language decoding models on the same dataset. This is limited by the data availability, since one needs a large amount of data that contain a wide range of words to train the model and this is the only dataset we know that fulfil our purpose and is large enough. To overcome this limitation, we performed cross-validation to test the generalizability of our model to new sentential contexts and to new subjects.

\subsection{Language predictors}

We include a variety of language predictors to decode ERPs from. As a baseline, we include three predictors (lexical frequency, lexical surprisal, semantic distance) that have previously been used in ERP analyses (Ettinger et al., 2016; Frank et al. 2015; Frank and Willems, 2017, Yan and Jaeger, 2019). Besides this, as an exploratory first step, we also examine two word embedding measures that has been used in a variety of natural language processing tasks (Pennington et al., 2014; Peters et al. 2018) but have not previously been used to predict ERPs.

Lexical frequency We use the same word frequency measurement used by (Frank and Willems, 2017). They use log-transformed (relative) word frequency in the COW14 corpus (Schäfer, 2015).

Lexical surprisal We use the lexical surprisal $-\log p\left(w_{i} \mid w_{1}, \ldots, w_{i-1}\right)$ obtained from a recurrent neural network that predicts upcoming words trained by Frank et al. (2015), shared by the first author of paper together with the EEG data.

Semantic distance Following Frank and Willems (2017), we point-wise average the GloVe embedding (Pennington et al., 2014) of each word prior to a particular word to obtain a context embedding and then calculate the cosine distance between the context embedding and the word embedding for that word. We use the GloVe embeddings trained on Wikipedia 2014 and Gigaword 5 (6B tokens, 400K vocabulary size).

While we used the same dataset and similar predictors as some previous studies (Frank and Willems, 2017), we note that the surprisal and semantic distance 
measures are different from what is used in Frank and Willems (2017). Specifically, they use the lexical surprisal trained on a n-gram model rather than RNN. They also train a word2vec model themselves and used it to compute the semantic distances.

Our measures differ from theirs because our goals are slightly different. They use the two measures they do because they aim to create measures of lexical surprisal and semantic distance that are minimally correlated to best delineate the contribution of each. In contrast, in the current study, we are more interested in including language predictors that capture as much linguistic information as possible, in order to provide a more robust baseline to test against. We thus choose measures that potentially captures richer information rather prioritizing de-correlating the contribution of each baseline predictor.

Static word embeddings The first word embedding measure we use is statici.e., it does not vary as a function of the context ${ }^{2}$ Specifically, we use GloVe word embeddings that have been shown to have good performance in a variety of natural language processing tasks (for details, see Pennington et al., 2014). We pass the word embeddings through a multi-layer perceptron with two hidden layers of hyperbolic tengent (tanh) non-linearities. The idea here is that the GloVe-only model tells us how much the distributional properties of a word, outside of the current context, contribute to ERPs.

Contextual word embeddings We also examine one contextual word embedding. As the name suggests, this type of word embedding also contains information about what context the word is embedded in. Contextual influence is typically exerted either through transformer modules (e.g., BERT, Devlin et al., 2018) or recurrent connections (e.g., ELMo, Peters et al., 2018). For the current study, we used contextual word embeddings generated from ELMo (Peters et al., 2018) using the allennlp package (Gardner et al., 2017). ELMo produces contextual word embeddings using a combination of character-level CNNs and bidirectional RNNs trained against a language modeling objective, and thus it is a useful contrast to GloVe, since it captures not only a word's distributional properties, but how they interact with the current context.

We take all three layers of the hidden layer output in the ELMo model and concatenate them. To ensure a fair comparison with the GloVe-based models, we use the same tuning procedure as for the static word embeddings. Further, because sentences are presented incrementally in ERP experiments and because ELMo contains bidirectional connections-i.e. words in the right context of a word will also affect the word embeddings of previous words-we do not obtain an embedding for a particular word on the basis of the entire sentence. Instead we use only the portion of the sentence up to and including that word to obtain its embedding to

\footnotetext{
${ }^{2}$ This type of word embedding models was considered state-of-the-art until 2018.
} 
keep parallel to incremental processing $3^{3}$

\subsection{Model training}

At train time, the decoder weights are frozen, and the encoder part is replaced by one or a combination of the language predictors described above. This entails fitting an interface mapping - a linear transformation for each channel produced by the encoder-from the features extracted from the language predictors into the representation space of the encoder ${ }^{4}$ As a baseline, we also train an intercept model that only has an intercept node (always has an activation of 1) linked to the encoder.

We examined all possible combinations of lexical frequency, lexical surprisal, semantic distance with one of the two word embeddings-i.e. no model contain both the static and the contextual word embedding. In total this creates 24 different combinations of the language predictors. We link the language predictors with each autoencoder architecture described in the previous session and examine the contribution of each language predictor.

All models are trained with mean squared error loss between the ERPs reconstructed from the language predictors and the actual ERPs over all electrodes- the same loss used for the CAE. Model weights are updated using mini-batch gradient descent with the same parameters used for the training of the autoencoder and each model is updated for 100 epochs in train time.

For the combined models, we perform a parameter search over the same three levels of weight decays $\left(10^{-1}, 10^{-3}, 10^{-5}\right)$ using 5 -fold cross-validation. We also adopted early stopping to further control for overfitting. As in the training of the autoencoder, we perform cross-validation both across subjects and across sentences. We use the same division of folds as used in the training of the autoencoder. For each fold, we use the weights of the corresponding autoencoder for the training of combined models.

\subsection{Model evaluation}

We evaluate the model performance in a variety of ways to examine the overall performance as well as the spatial-temporal patterns of the decoding ability of the model.

\footnotetext{
${ }^{3}$ We note that using word embeddings trained with bi-directional LSTM to approximate incremental processing can be sub-optimal. When we ran this project, there was no contextualized word embeddings that only take information from previous context. This has changed with the development of OpenAI GPT-2 (Radford et al. 2019). We return to this point in Section 4 where we discuss future directions.

${ }^{4}$ The codes used for training the models can be found here
} 


\subsubsection{Overall performance}

We use two metrics to evaluate the overall performance of the combined models. First, we quantify the approximate amount of variance explained by each model using a modified version of the $R^{2}$ measure to account for the fact that different autoencoders have different baseline performance to start with. Specifically, we use the following formula:

$$
R_{\text {mod }}^{2}=1-\frac{\mathrm{MSE}_{\text {model }}-\mathrm{MSE}_{\mathrm{CAE}}}{\mathrm{MSE}_{\text {intercept }}-\mathrm{MSE}_{\mathrm{CAE}}}
$$

Here we consider the performance of the autoencoder as an upper bound for the performance of the combined model and the intercept model serves as a lower bound. The modified $R^{2}$ measure thus represents how much improvement each language predictor model incurs with regard to the performance of the autoencoder. This metric provides an intuitive measure of how much of the data is explained by each model.

We also examine the Pearson correlation between the decoded ERPs and the actual ERPs. For each trial of the development set, we calculated the Pearson correlation between the decoded ERPs and the actual ERPs across all 200 time points and all 32 electrodes. We then run a linear mixed-effects model over the trial-level correlation from each combined model to evaluate the contribution of each language predictor in decoding the ERPs.

For each model, if a language predictor is included, the corresponding predictor is coded as 1; otherwise, it is coded as 0. Previous models found that ERPs of function words are overall less decodable than content words (Frank et al., 2015). Informed by such findings, we also included a predictor for word type (content vs. function words). Word types are tagged using the spaCy package (Honnibal and Montani, 2017). Content words are coded as 1 and function words are coded as 0.5 To examine whether different predictors affect each other, we also included interaction between different language predictors. The rationale behind this is that, if two predictors have a negative interaction, it suggests that they explain overlapping parts of the variance, and thus, when both are included in the model, the overall contribution is smaller than the sum of the contribution of each language predictor when it is included by itself. On the other hand, positive interaction would indicate that the combination of these two predictors can explain ERPs better than each predictor on its own.

\subsubsection{Spatial temporal performance}

To examine at what time and at what scalp locations the language models improve the decoding of ERPs, we calculate the model performance at different time win-

\footnotetext{
${ }^{5}$ For this analysis, we excluded any words that contain any form of contraction, e.g., its, and thus can potentially contain both content and function words. We considered words with the tags ADP, CCONJ, DET, NUM, PART, PRON as function words and words with the tags ADJ, ADV, NOUN, PROPN, VERB as content words.
} 
dows on each electrode. Specifically, we use a moving window with a length of $80 \mathrm{~ms}$ and stride size of $40 \mathrm{~ms}$, and calculate, within each time window, the correlation across time points between the decoded ERPs and the actual ERPs at each electrode. We then examine whether and how the inclusion of different predictors increase the correlations.

We focus on two sets of comparisons. First, we calculate the correlation metrics for models that only include each language predictor by itself, and compared it to the intercept model to examine the improvement in decoding performance of each language predictor compared to the intercept. Second, we zoom into the additional contribution of the two word embedding predictors and examine whether and how the performance of each model performance changes when including either word embedding measure on top of the three monolithic predictors.

To illustrate the temporal-spatial profile of the model performances, we create topographical plots that showcase the scalp distribution of the increase in correlations within each time window. The plots are generated through interpolation using codes modified from Craddock (2017). Specifically, we first place the correlation value at the corresponding scalp location and then interpolated values to create contour plots of where the model performances are improved by the inclusion of a language predictor.

\subsection{Overall performance}

Next, we examine the models that decode ERPs from language predictors using our pre-trained autoencoder. Since the wide models overall have better performance than the narrow models, we use the wide models for all our combined models. In the following part, we first report the overall performance of each autoencoder when combined with language predictors. Then, we focus in on the autoencoder that gives the best performance-specifically, the alpha autoencoder-and examine the spatial-temporal profile of this model's performance.

Baseline performance First, we examine the performance of the baseline model, where only an intercept term with an activation of 1 is linked to the trained autoencoder. The mean squared errors (MSE) for each of the model is shown in the Figure 6 plotted along with the variance of the dataset-i.e. the MSE of a model that predicts the mean of the dataset. As can be seen, while the other three models have MSEs that are similar to the variance of the dataset, the theta model has MSEs that are a lot larger than the variance. This shows that the theta model with just an intercept cannot approximate the mean trend of the data well. In other words, it performs a lot worse than just predicting the mean of the dataset. This suggests the trained theta model is rather inflexible.

In contrast, the other three models are largely able to reconstruct the general trend of the data. Of the other three models, the beta and gamma models have MSEs that are almost the same as the VAR of the dataset. The alpha model, on the other hand, shows middling performance. Noticeably, the MSE is much smaller 


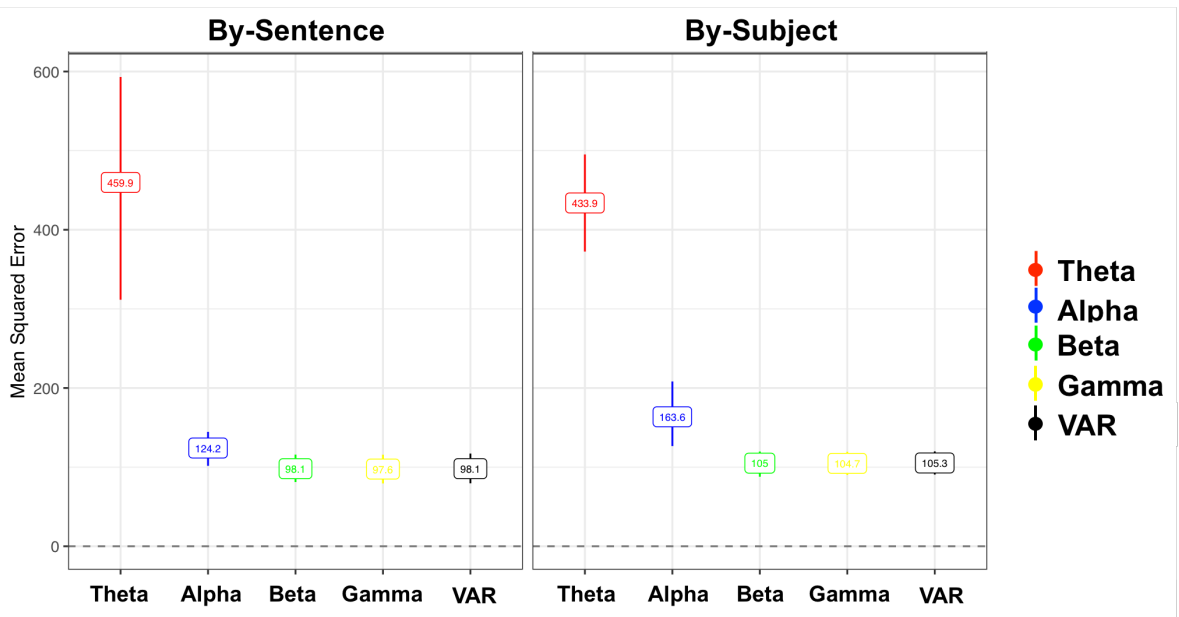

Figure 6: Decoding performance of the intercept only model for each autoencoder. Numbers represent mean squared errors across folds. Lines represent bootstrapped confidence intervals.

when cross-validating across sentences than across subjects, suggesting that the mean trend learned by the alpha model is better generalizable across sentences than across subjects.

Single predictor models Next, we examine how much each predictor improves the model performance. We compare the performance of each model using the modified $R^{2}$ metrics as described in Section 3.5 . The results are presented in Figure 7. An $R^{2}$ larger than 0 would indicate that the inclusion of a predictor improves the model's performance in decoding ERPs and it can be generalized from the training set to the test set (since the results are from cross-validation). One the other hand, an $R^{2}$ equal to or even smaller than zero would indicate that the pattern on the training set cannot be generalized to the test set.

There are several patterns worth mentioning. First, while the beta and gamma models perform the best in terms of autoencoder reproductibility, they do not perform the best when used to reconstruct ERPs from language predictors. This is potentially because the trained autoencoders are over-entrained to the variability in the ERPs that are unrelated to the language signal. On the other hand, the theta model and alpha model show better decoding performance when paired with language predictors, possibly because the encoded states reflect signals in the data that are more task relevant.

Second, how well each predictor generalize across subjects and items differ slightly with regard to whether the cross validation was done across subjects or items. For example, the theta models seem to overall have better performance when cross-validated across subjects. It is possible that when training across subjects, the theta model is overfitted to the subject-specific information in the lower frequency 


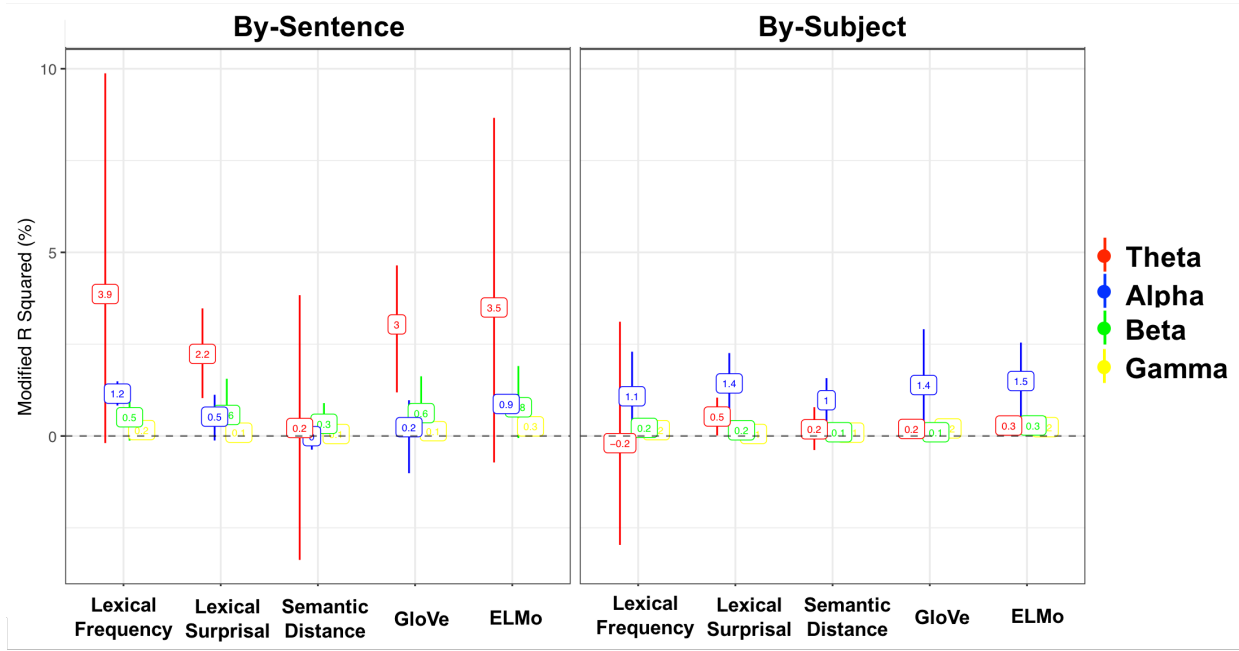

Figure 7: Decoding performance of the model when each language predictor is added to the intercept model. Numbers represent modified $R^{2}$. Lines represent bootstrapped confidence intervals.

range hence the generalizability is not as good.

Lastly, although the theta model overall has larger improvement with regard to the baseline (as shown above), the performances of the baseline theta models are a lot worse than the other models. Thus, the best-performing model among the four structures we examined is the alpha model.

All-in models Next we zoom into the contribution of each word embeddings measure. To do this, we use the model that includes all the predictors (lexical frequency, lexical surprisal and semantic distance), comparing it against the model that has word embeddings on top of it. As shown in Figure 8, while including word embeddings by themselves can improve model performance, adding word embeddings on top of all the language predictors does not further increase the overall performance.

In the following parts, we focus on the best-performing model and run some more detailed diagnoses on the models using the alpha autodecoder.

\subsubsection{Linear mixed-effects models}

We run linear mixed-effects models over the models that include all possible combinations of language predictors to evaluate the contribution of each prediction (see Methods for detail).

Across-sentences Table 3 shows the analyses on performances of the model when doing cross-validation across sentences. With regard to the contribution 


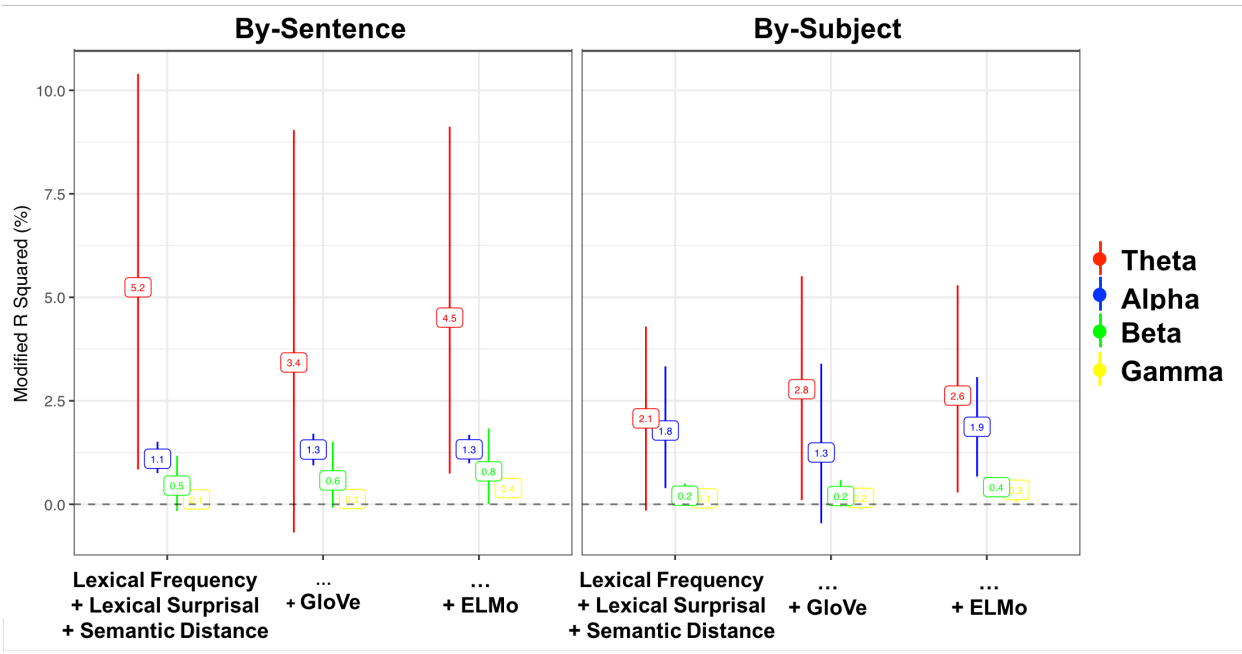

Figure 8: Decoding performance of the model when adding each word embedding measure to a model already containing all three monolithic langauge predictors. Numbers represent modified $R^{2}$. Lines represent bootstrapped confidence intervals.

of each language predictor, there is a significant effect of lexical frequency $(t=$ $3.68, p<0.001)$, lexical surprisal $(t=3.10, p<0.05)$, and semantic distance $(t=$ $3.76, p<0.001$ ), indicating that the inclusion of each predictor increases the model's ability to decode ERPs.

As for the two word embedding measures, while including ELMo increases model performance $(t=2.63, p<0.01)$, including GloVe does not significantly increase the performance of the model $(t=-0.11, n$.s. $)$. This suggests that information the models extract from ELMo word embeddings can be generalized across language materials in decoding ERPs.

In terms of two way interactions, there is a significant significant negative interaction between lexical frequency and lexical surprisal $(t=-4.99, p<0.001)$ and between lexical surprisal and ELMo $(t=-1.96, p<0.05)$ and a marginally significant negative interaction between lexical frequency and semantic distance $(t=-1.68, p=0.09)$, suggesting that these predictors contain overlapping information that is related to ERP signals. No other two-way interactions between language predictors were reliable.

Finally, word type has a positive effect $(t=3.10, p<0.002)$, indicating that the models are better at decoding content words than function words. This is consistent with with previous findings (Frank et al., 2015).

\begin{tabular}{cllll}
\hline Predictor & $\hat{\beta}$ & $\mathrm{t}$ & \\
\hline Intercept & 0.008 & 0.52 & & \\
Word Type (Content) & 0.008 & 3.10 & ${ }^{* *}$ \\
Lexical Frequency & 0.004 & 3.68 & ${ }^{* *}$
\end{tabular}




\begin{tabular}{crrll}
\hline Predictor & \multicolumn{1}{c}{$\hat{\beta}$} & \multicolumn{1}{c}{$\mathrm{t}$} & \\
\hline Lexical Surprisal & 0.003 & 3.11 & ${ }^{* *}$ \\
Semantic Distance & 0.004 & 3.76 & $* *$ \\
GloVe & -0.0001 & -0.11 & \\
ELMo & 0.003 & 2.63 & $* *$ \\
Freq: Word Type & 0.001 & 1.29 & \\
Surp: Word Type & -0.0001 & -0.11 & \\
SemDis: Word Type & -0.0003 & -0.33 & \\
Glove: Word Type & -0.0003 & -0.29 & \\
ELMo: Word Type & -0.0002 & -0.24 & \\
Freq: Surp & -0.004 & -4.99 & $* *$ \\
Freq: SemDis & -0.001 & -1.68 & + \\
Freq: GloVe & 0.001 & 0.92 & \\
Freq: ELMo & -0.001 & -1.27 & \\
Surp: SemDis & -0.001 & -1.38 & \\
Surp: GloVe & 0.001 & 0.79 & \\
Surp: ELMo & -0.002 & -1.96 & $*$ \\
SemDis: GloVe & -0.0003 & -0.27 & \\
SemDis: ELMo & 0.0005 & 0.54 & \\
\hline
\end{tabular}

Table 3: Model estimates and $\mathrm{t}$ statistics from the mixed-effects model on the contribution each language predictor to the decoding performance of the model that uses the alpha autoencoder. Model performance is measured by correlation between decoded ERPs and original ERPs, cross-validated across sentences. ${ }^{* *}: p<0.01$; ${ }^{*}: p<0.05 ;^{+}: p<0.1$

Across-subjects Table 4 shows the performance of the models cross-validated across subjects. With regard to the contribution of each language predictor, there is a significant effect of lexical frequency $(t=4.62, p<0.001)$ and a marginal effect of surprisal $(t=1.92, p=0.054)$. The effect of semantic distance does not reach significance $(t=-1.44, n . s$.). As for the two word embedding measures, including GloVe increases model performance $(t=2.43, p<0.05)$, but including ELMo does not significantly increase the performance of the model $(t=-0.35, n . s$. $)$. This suggests that information the models extract from GloVe word embeddings can be generalized across subjects in decoding ERPs.

In terms of two way interactions, there is a significant negative interaction between lexical frequency and lexical surprisal $(t=-2.55, p<0.05)$ and between lexical frequency and GLoVe $(t=-4.28, p<0.001)$ and a marginally significant negative interaction between lexical frequency and ELMo $(t=-1.79, p=0.07)$ and between semantic distance and GLoVe $(t=-1.76, p=0.08)$, suggesting that 
these predictors explain shared variances in the model. There is a significant positive interaction between lexical frequency and semantic distance $(t=2.97, p<$ 0.01 ), suggesting that the two factors when both included explained more variance than each predictor by itself. No other two-way interactions between language interactions reached significance. The effect of word type does not reach significance $(t=-0.81$, n.s. $)$ nor does its interaction with any of the language predictor.

\begin{tabular}{crrll}
\hline Predictor & \multicolumn{1}{c}{$\hat{\beta}$} & \multicolumn{1}{c}{$\mathrm{t}$} & \\
\hline Intercept & -0.013 & -0.84 & \\
Word Type (Content) & -0.002 & -0.81 & \\
Lexical Frequency & 0.005 & 4.62 & $* *$ \\
Lexical Surprisal & 0.002 & 1.92 & + \\
Semantic Distance & -0.001 & -1.42 & \\
GloVe & 0.003 & 2.43 & $*$ \\
ELMo & -0.0004 & -0.35 & \\
Freq: Word Type & -0.001 & -0.71 & \\
Surp: Word Type & -0.0003 & -0.33 & \\
SemDis: Word Type & 0.0001 & 0.15 & \\
Glove: Word Type & 0.001 & 0.53 & \\
ELMo: Word Type & -0.0002 & -0.19 & \\
Freq: Surp & -0.002 & -2.55 & $*$ \\
Freq: SemDis & 0.002 & 2.97 & $* *$ \\
Freq: GloVe & -0.004 & -4.28 & $* *$ \\
Freq: ELMo & -0.002 & -1.79 & + \\
Surp: SemDis & -0.001 & -1.16 & \\
Surp: GloVe & 0.001 & 0.82 & \\
Surp: ELMo & 0.001 & 1.02 & \\
SemDis: GloVe & -0.002 & -1.76 & + \\
SemDis: ELMo & 0.0003 & 0.27 &
\end{tabular}

Table 4: Model estimates and $\mathrm{t}$ statistics from the mixed-effects model on the contribution each language predictor to the decoding performance of the model that uses the alpha autoencoder. Model performance is measured by correlation between decoded ERPs and original ERPs, cross-validated across subjects. ${ }^{* *}: p<0.01$; ${ }^{*}: p<0.05 ;^{+}: p<0.1$

\subsection{Spatial-temporal signature of model performance}

In the following part, we examine when and where on the scalp each language predictor improves the model's ability to decode ERPs (see Section 3.5 for details). 
In the same fashion as above, we first examine the effect of each language predictor by itself (single predictor models) and then examine the effect of the two word embedding measures on top of all the language predictors (all-in models). We also present the results both for models cross-validated across sentences and subjects. When the inclusion of a predictor increases the model's decoding performance for a certain time window and for certain electrodes, we consider that this predictor has an effect for that time window and location.

\subsubsection{Single predictor models}

\section{Across-sentences}

Lexical frequency Lexical frequency shows an effect as early as $\sim 100 \mathrm{~ms}$ after word onset, and the effect is bi-lateral and frontal. This effect lies within the N1/P1 time window, consistent with previous findings (Yan and Jaeger, 2019; Hauk et al., 2006, Laszlo and Federmeier, 2014). There is also an effect at $180 \mathrm{~ms}$. In this time window, besides a bi-lateral, frontal effect, there is also an effect that is right-lateralized and centro-parietally distributed. In later time windows, there is a centro-parietal effect at $\sim 420 \mathrm{~ms}$, which is slightly right-lateralized. This effect is followed by an effect at $\sim 540 \mathrm{~ms}$, but this time more frontally distributed. This pattern agrees with previous findings using more conventional analysis methods (Hauk et al., 2006; Yan and Jaeger, 2019).

Lexical surprisal Lexical surprisal shows an effect as early as $\sim 140 \mathrm{~ms}$ after word onset, the effect is left-lateralized and posterior. This effect lies within the N1/P1 time window, consistent with previous findings (Yan and Jaeger. 2019). There is also an effect at $\sim 260 \mathrm{~ms}$, this effect is centro-frontally distributed, possibly corresponding to a N250 effect (Grainger et al., 2006). This pattern is also consistent with previous findings (Frank and Willems, 2017; Yan and Jaeger, 2019). In later time windows, there is a small frontal effect at $\sim 380 \mathrm{~ms}$ followed by a posterior effect at $\sim 420 \mathrm{~ms}$. There is also a small frontal effect at $\sim 500 \mathrm{~ms}$. This pattern agrees with previous findings using more conventional analysis methods (Yan and Jaeger, 2019, Frank and Willems, 2017).

Semantic distance Semantic distance shows an effect as early as $\sim 100 \mathrm{~ms}$ after word onset, the effect is bi-lateralized and frontal, followed by a bi-lateral, posterior effect $\sim 140-180 \mathrm{~ms}$. This effect lies within the N1/P1 time window, consistent with previous findings (Yan and Jaeger, 2019). There is also a centro-frontal effect at $\sim 260 \mathrm{~ms}-$ possibly corresponding to an N250 effect. This pattern is consistent with previous findings (Yan and Jaeger, 2019, Frank and Willems, 2017). In later time windows, there is a centro-parietal effect at $\sim 420 \mathrm{~ms}$ and a centro-frontal effect $\sim 500 \mathrm{~ms}$. This pattern agrees with previous findings using more conventional analysis methods (Yan and Jaeger, 2019; Frank and Willems, 2017). 


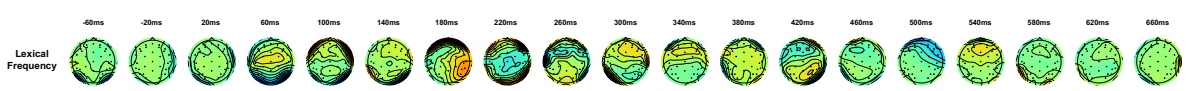 $=0000000000000000000$ $=0000000000000000000 \mathbf{E}$ $-0000000000000000000$ $-0000000000000000000$}

Figure 9: Increase in correlation between decoded ERPs and actual ERPs when including each language predictor in the model in different time windows at different scalp locations. Cross-validated across sentences.

GloVe GloVe embeddings has an effect as early as $\sim 100 \mathrm{~ms}$ after word onset, the effect is right-lateralized and frontal, followed by a bi-lateral, posterior effect $\sim 140-180 \mathrm{~ms}$. This effect lies within the N1/P1 time window. There is also a centro-frontal effect at $260 \mathrm{~ms}$, possibly corresponding to a N250 effect. In later time windows, there is a small centro-parietal effect at $420 \mathrm{~ms}$ and a small centrofrontal effect at $\sim 500 \mathrm{~ms}$. The pattern looks similar to that of surprisal and semantic distance but is overall smaller.

ELMo ELMo features has an effect as early as $~ 140-180 \mathrm{~ms}$ after word onset, the effect is bi-lateralized and posterior. This effect lies within the N1/P1 time window. There is also a centro-frontal effect at $\sim 260 \mathrm{~ms}$, likely corresponding to a $\mathrm{N} 250$ effect. In later time windows, there is a small centro-frontal effect at $\sim 380 \mathrm{~ms}$ and a small centro-frontal effect at $\sim 500 \mathrm{~ms}$. The pattern again looks similar to that of surprisal and semantic distance but is overall smaller.

\section{Across-subjects}

Lexical frequency Lexical frequency has an effect as early as $\sim 60-100 \mathrm{~ms}$ after word onset. This effect is bi-lateral and posterior. There is a bi-lateral, posterior effect $\sim 180-260 \mathrm{~ms}$. This effect is followed by an effect with similar topo-graphical distribution at $340 \mathrm{~ms}$. In later time windows, there is a bi-lateral, posterior effect $540 \mathrm{~ms}$.

Lexical surprisal Lexical surprisal has an effect as early as $\sim 60 \mathrm{~ms}$ after word onset. This effect is bi-lateral and posterior. Starting $\sim 100 \mathrm{~ms}$ there also seems to be a more centro-parietal effect. There also seems to be a bi-lateral, frontal effect $\sim 340-380 \mathrm{~ms}$. 


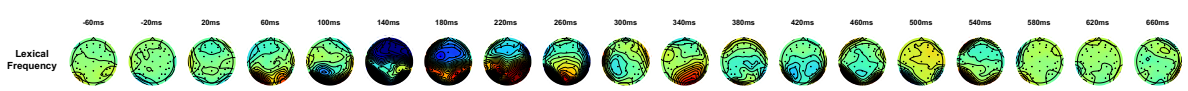 $=0000000000000000000$ $=0000000000000000000 \mathrm{E}$ $-0000000000000000000$ $-0000000000000000000$}

Figure 10: Increase in correlation between decoded ERPs and actual ERPs when including each language predictor in the model in different time windows at different scalp locations. Cross-validated across subjects.

Semantic distance Semantic distance has an effect as early as $\sim 60-100 \mathrm{~ms}$ after word onset. This effect is bi-lateral and posterior. Starting $\sim 140 \mathrm{~ms}$ there also seems to be a more centro-parietal effect. There a left-lateralized, posterior effect at $\sim 340 \mathrm{~ms}$. In later time windows, there is a small frontal effect at $\sim 500 \mathrm{~ms}$.

GloVe GloVe has an effect as early as $\sim 60-100 \mathrm{~ms}$ after word onset. This effect is bi-lateral and posterior. Starting $\sim 140 \mathrm{~ms}$ there also seems to be a more centro-frontal effect. There a left-lateralized, centro-parietal effect at $\sim 340 \mathrm{~ms}$.

ELMo ELMo has an effect as early as $\sim 100 \mathrm{~ms}$ after word onset. This effect is bi-lateral and posterior. At $\sim 140 \mathrm{~ms}$ there also seems to be a more centro-frontal effect.

\subsubsection{All-in Models}

Next, we zoom in on the performance of the word embedding predictors and test whether and how they improve model performance over the three monolithic measures (lexical frequency, lexical surprisal, and semantic distance).

\section{Across-sentences}

GloVe GloVe seems to have a small effect as early as $\sim 180 \mathrm{~ms}$ after word onset. This effect is centro-frontally distributed.

ELMo ELMo seems to have a small effect as early as $\sim 180 \mathrm{~ms}$ after word onset. This effect is right-lateralized and centrally distributed. In later windows, there also seems to be a left-lateralized, posterior effect at $\sim 500 \mathrm{~ms}$.

\section{Across-subjects}




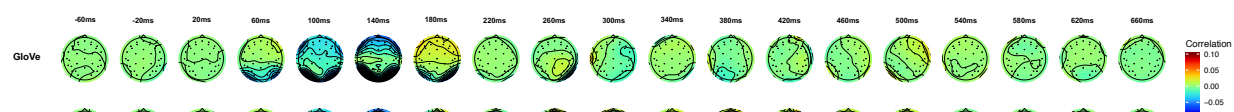 $-000000000000000000000^{*}$}

Figure 11: Increase in correlation between decoded ERPs and actual ERPs when including each word embedding predictor on top of the monolithic predictors (lexical frequency, lexical surprisal, and semantic distance) in different time windows at different scalp locations. Cross-validated across sentences.

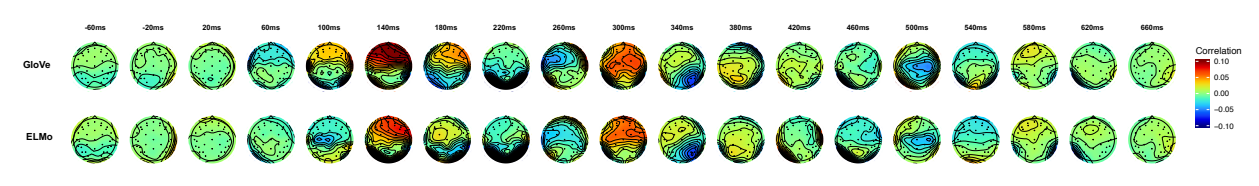

Figure 12: Increase in correlation between decoded ERPs and actual ERPs when including each word embedding predictor on top of the monolithic predictors (lexical frequency, lexical surprisal, and semantic distance) in different time windows at different scalp locations. Cross-validated across subjects.

GloVe GloVe has an effect as early as $\sim 100-180$ ms after word onset. This effect is centro-frontally distributed. This is followed by an effect at $\sim 300 \mathrm{~ms}$ that has similar topographical distribution. In later time windows, there seems to be a small effect at $\sim 540 \mathrm{~ms}$. This effect is posteriorlly distributed, and followed by a frontal effect at $\sim 580 \mathrm{~ms}$.

ELMo ELMo has an effect as early as $\sim 140-180 \mathrm{~ms}$ after word onset. This effect is centro-frontally distributed. This is followed by an effect at $\sim 300 \mathrm{~ms}$ that has similar topographical distribution. In later time windows, there is a leftlateralized, posterior effect $\sim 420-460 \mathrm{~ms}$. Similar to the effect of GloVe features, there also seems to be a small effect at $\sim 540 \mathrm{~ms}$. This effect is posteriorly distributed, and followed by a frontal effect at $\sim 580 \mathrm{~ms}$.

\subsection{Discussion}

We show that our framework is able to replicate classic findings using more conventional methods (e.g., Yan and Jaeger, 2019, Frank et al., 2015, Hauk et al., 2006), validating this new framework as a promising new tool for exploratory research. At the same time, we explore the ERP correlates associated with word embeddings, and found that the pattern looks very similar to lexical surprisal and semantic distance. When adding word embeddings to a model that already contains lexical surprisal and semantic distance, however, word embeddings do not seem to explain much additional variances of the data. At first sight, it might suggest that it is unnecessary to use this more complicated measure to model ERPs, but this could also due to the limits of the current study and requires further investigation. 
This study also reveals two stark differences between the results of the autoencoders and the combined models. First, the less compressed autoencoders overall perform better than more compressed autoencoder. This is unsurprising because these models are less compressed and can potentially encode both higher and lower frequency signals. However, while less compressed autoencoders overall have better reconstruction performance, they do not perform better in revealing the ERP correlates of language processing. Instead, the alpha model seems to perform overall the best in modeling language effects, followed by the theta model. This is possibly because the ERP correlates of language processing have similar duration as the time step size of our alpha and theta models. The beta and gamma models, on the other hand, might focus on maximizing the correlation with actual ERPs by matching with faster-changing effects that does not relate to language processing and consequently does not perform as well in detecting language effects.

Second, while all the autoencoders show little difference when they are crossvalidated across subjects or items, the combined models show much bigger sensitivity to the cross-validation scheme. Overall it seems that the alpha models cross-validated with items produces the best overall performance and also creates similar spatial-temporal signatures as previous findings. The performance of the same model, however, was a lot worse when trained and evaluated across subjects. This potentially indicate that certain patterns of language predictor effects captured by the alpha model are more generalizable across items than they do across subjects. This is not that surprising given that there is large individual differences in ERPs (Polich, 1997).

Our results also inform our understanding about what information is contained in word embeddings. Previous ERP studies mainly used word embeddings to calculate semantic distance (Broderick et al., 2018; Frank and Willems, 2017; Yan and Jaeger, 2019; Ettinger et al., 2016), the current study complement these studies and directly examined the information contained in word embeddings. While including word embeddings can improve the model's performance in decoding ERPs, when scrutinizing where these improvements occur, they do not always show up in time windows associated with higher level processing - e.g. the N400 Kutas and Hillyard, 1980) and P600(Osterhout and Holcomb, 1992). When including only the word embeddings in the model, they affect both components related semantic processing - e.g. the N400 - and early components that are related to perceptual (e.g. the N1/P2 Hauk et al., 2006, Laszlo and Federmeier, 2014) and orthographic processing (N250 Grainger et al., 2006). This is worth noting because word embeddings are oftentimes considered to reflect semantic information while our results indicate that it might also capture orthographic or perceptual information. This provides new insight into how one should interpret NLP results using word embeddings. On the other hand, this pattern is very similar to that of lexico-semantic predictors (e.g. lexical surprisal, semantic distance Yan and Jaeger, 2019). Therefore, this pattern could also be a showcasing top-down influence of higher-level information to lower-level processing (see, e.g., Kim and Lai, 2012; Dikker et al., 2010; Dambacher et al., 2006). 
Lastly, we do not see qualitative differences in performance for contextualized (ELMo) and Glove features. When analyzing the overall effects of language predictors using mixed-effects model, the effect of ELMO is significant when doing cross-validation across sentences, while the effects of GloVe is significant when doing cross-validation across subjects. This could potentially mean that contextualized word embeddings are better at generalizing across different sentential contexts.

While the contextualized word embeddings do not seem to have a clear advantage over static word embedding in decoding ERPs, this could partly be caused by how contextualized word embeddings are generated. To simulate incremental processing, we fed in sentence segments of the sentences up until the critical word to generate ELMo embeddings. However, ELMo models were trained using entire sentences with bi-directional LSTM connections. This way of using ELMo model might not be optimal given how the model was originally trained. This issue might be circumvented with more recently developed word embeddings, e.g., OpenAI GPT-2 (Radford et al., 2019), where only the context to the left of the word can affect the computation of the contextualized word embeddings. Future research is required to examine whether these types of contextualized word embeddings can more in decodng ERPs.

\section{Conclusion and Future Directions}

In the current paper, we propose a novel framework for modeling ERPs in sentence reading. We show that our models are able to replicate existing findings using more conventional analysis methods (Hauk et al., 2006; Frank and Willems, 2017; Yan and Jaeger, 2019; Laszlo and Federmeier, 2014), validating the new framework's ability to find ERP correlates of language processing. Working the other way round, our model can also help probe into the representations constructed from NLP research. We provide one example of how such probing can be carried out and examine what information is encoded in word embeddings. We find that word embeddings predict similar ERP patterns as lexical surprisal and semantic distance. These results show that the proposed framework is promising in flexibly linking language models to ERPs, and can be used for exploratory analyses in situations where one does not want make strong assumptions about when, where, and how the language predictors affect ERPs.

In the current study, we train the autoencoders and the language decoding models on the same dataset. As explained in Section 3, this is due to the type and size of the dataset what need to train the model. With the growing interest in exploring ERPs of processing naturalistic linguistic stimuli, there will hopefully be more datasets that can be used to train models. In the future, one can potentially further improve the robustness of the model by aggregating datasets to form a large training set for an autoencoder that can be further fine tuned to decode ERPs within a simple experiment. 
One challenge that comes with training and testing across ERP datasets is that different datasets might have different electrode locations and different number of electrodes. For many conventional analysis, this comprises an issue when generalizing the results from one dataset to another, e.g., if one analyzes the effects on individual electrodes (e.g., Laszlo and Federmeier, 2014; Frank and Willems, 2017; Yan and Jaeger, 2019) or the effects aggregated across a selected set of electrodes (e.g., Dambacher et al., 2006, Frank et al., 2015; Yan and Jaeger, 2019). For our approach, however, this is less of a issue. In fact, this is precisely why we include a fully mixed layers before passing the ERP data through any convolutional layers (and the same structure on the decoder side). This layer allows all the electrodes to freely combine with each other to unveil underlying processes that give rise to the activation pattern of the electrodes. This means that, when applying a pre-trained autoencoder to a new dataset, one can swap out these fully connected layers and fine tune the connections within the dataset it applied to. While all our results are cross-validated, they come from the same dataset with the same recorded electrodes. It remains to be tested whether the model can indeed be generalizable to datasets that use different recording schemes.

The current model is agnostic to what computation underlies language processing. In other words, it tests what information is used at what stage of processing without explicitly specifying the underlying computation. However, this could be done by swapping the language model attached to the ERP decoder. Instead of using word embeddings, one could instead use computational models that are designed to explicitly reflect what cognitive processes underlie language processing (e.g., Brouwer et al., 2017; Rabovsky et al., 2018). Future work needs to be done to test how well this framework can provide support for different accounts of language processing.

\section{References}

Stanislaw Antol, Aishwarya Agrawal, Jiasen Lu, Margaret Mitchell, Dhruv Batra, C Lawrence Zitnick, and Devi Parikh. VQA: Visual Question Answering. In Proceedings of the IEEE international conference on computer vision, pages 2425-2433, 2015.

Yoshua Bengio, Aaron Courville, and Pascal Vincent. Representation learning: A review and new perspectives. IEEE transactions on pattern analysis and machine intelligence, 35(8):1798-1828, 2013.

R Clifford Blair and Walt Karniski. An alternative method for significance testing of waveform difference potentials. Psychophysiology, 30(5):518-524, 1993.

Michael P Broderick, Andrew J Anderson, Giovanni M Di Liberto, Michael J Crosse, and Edmund C Lalor. Electrophysiological correlates of semantic dissimilarity reflect the comprehension of natural, narrative speech. Current Biology, 28(5):803-809, 2018. 
Harm Brouwer, Matthew W Crocker, Noortje J Venhuizen, and John CJ Hoeks. A neurocomputational model of the N400 and the P600 in language processing. Cognitive Science, 41:1318-1352, 2017.

Matt Craddock. ERP Visualization: Creating topographical scalp maps: part 1, Feb 2017. URL https://www.mattcraddock.com/blog/2017/02/25/ erp-visualization-creating-topographical-scalp-maps-part-1/ Accessed: 2019-05-30.

Michael Dambacher, Reinhold Kliegl, Markus Hofmann, and Arthur M Jacobs. Frequency and predictability effects on event-related potentials during reading. Brain Research, 1084(1):89-103, 2006.

Katherine A DeLong, Thomas P Urbach, and Marta Kutas. Probabilistic word preactivation during language comprehension inferred from electrical brain activity. Nature Neuroscience, 8(8):1117-1121, 2005.

Jacob Devlin, Ming-Wei Chang, Kenton Lee, and Kristina Toutanova. Bert: Pretraining of deep bidirectional transformers for language understanding. ArXiv, 2018. URL https://arxiv.org/abs/1810.04805.

Suzanne Dikker, Hugh Rabagliati, Thomas A Farmer, and Liina Pylkkänen. Early occipital sensitivity to syntactic category is based on form typicality. Psychological Science, 21(5):629-634, 2010.

Allyson Ettinger, Naomi Feldman, Philip Resnik, and Colin Phillips. Modeling N400 amplitude using vector space models of word representation. In Proceedings of the 38th Annual Conference of the Cognitive Science Society, pages 1445-1450, 2016.

Kara D Federmeier and Marta Kutas. A rose by any other name: Long-term memory structure and sentence processing. Journal of memory and Language, 41(4): 469-495, 1999.

Stefan L Frank and Roel M Willems. Word predictability and semantic similarity show distinct patterns of brain activity during language comprehension. Language, Cognition and Neuroscience, 32(9):1192-1203, 2017.

Stefan L Frank, Leun J Otten, Giulia Galli, and Gabriella Vigliocco. The ERP response to the amount of information conveyed by words in sentences. Brain and Language, 140:1-11, 2015.

Matt Gardner, Joel Grus, Mark Neumann, Oyvind Tafjord, Pradeep Dasigi, Nelson F. Liu, Matthew Peters, Michael Schmitz, and Luke S. Zettlemoyer. AllenNLP: A Deep Semantic Natural Language Processing Platform. 2017.

Andrew Gelman and Eric Loken. The statistical crisis in science. The best writing on mathematics, 102(6):460-465, 2014. 
Jonathan Grainger, Kristi Kiyonaga, and Phillip J Holcomb. The time course of orthographic and phonological code activation. Psychological Science, 17(12): 1021-1026, 2006.

Peter Hagoort, Lea Hald, Marcel Bastiaansen, and Karl Magnus Petersson. Integration of word meaning and world knowledge in language comprehension. Science, 304(5669):438-441, 2004.

John Hale, Chris Dyer, Adhiguna Kuncoro, and Jonathan Brennan. Finding syntax in human encephalography with beam search. In Proceedings of the 56th Annual Meeting of the Association for Computational Linguistics (Volume 1: Long Papers), pages 2727-2736. Association for Computational Linguistics, 2018. URL http://aclweb.org/anthology/P18-1254.

Olaf Hauk, Matthew H Davis, M Ford, Friedemann Pulvermüller, and William D Marslen-Wilson. The time course of visual word recognition as revealed by linear regression analysis of ERP data. Neuroimage, 30(4):1383-1400, 2006.

Geoffrey E Hinton and Richard S Zemel. Autoencoders, minimum description length and helmholtz free energy. In Advances in neural information processing systems, pages 3-10, 1994.

Matthew Honnibal and Ines Montani. spacy 2: Natural language understanding with bloom embeddings, convolutional neural networks and incremental parsing. To appear, 2017.

MD. Zakir Hossain, Ferdous Sohel, Mohd Fairuz Shiratuddin, and Hamid Laga. A comprehensive survey of Deep Learning for Image Captioning. ACM Comput. Surv., 51(6):118:1-118:36, February 2019. ISSN 0360-0300.

Andrej Karpathy, George Toderici, Sanketh Shetty, Thomas Leung, Rahul Sukthankar, and Li Fei-Fei. Large-scale video classification with convolutional neural networks. In Proceedings of the IEEE conference on Computer Vision and Pattern Recognition, pages 1725-1732, 2014.

Albert Kim and Vicky Lai. Rapid interactions between lexical semantic and word form analysis during word recognition in context: Evidence from erps. Journal of cognitive neuroscience, 24(5):1104-1112, 2012.

Albert Kim and Lee Osterhout. The independence of combinatory semantic processing: Evidence from event-related potentials. Journal of Memory and Language, 52(2):205-225, 2005.

Yoon Kim. Convolutional neural networks for sentence classification. arXiv preprint arXiv:1408.5882, 2014.

Alex Krizhevsky, Ilya Sutskever, and Geoffrey E Hinton. Imagenet classification with deep convolutional neural networks. In Advances in neural information processing systems, pages 1097-1105, 2012. 
Gina R Kuperberg. Separate streams or probabilistic inference? what the N400 can tell us about the comprehension of events. Language, Cognition and Neuroscience, 31(5):602-616, 2016.

Gina R Kuperberg, Tatiana Sitnikova, David Caplan, and Phillip J Holcomb. Electrophysiological distinctions in processing conceptual relationships within simple sentences. Cognitive Brain Research, 17(1):117-129, 2003.

Marta Kutas and Kara D Federmeier. Thirty years and counting: finding meaning in the $\mathrm{n} 400$ component of the event-related brain potential (ERP). Annual review of psychology, 62:621-647, 2011.

Marta Kutas and Steven A Hillyard. Reading senseless sentences: Brain potentials reflect semantic incongruity. Science, 207(4427):203-205, 1980.

Sarah Laszlo and Kara D Federmeier. Never seem to find the time: evaluating the physiological time course of visual word recognition with regression analysis of single-item event-related potentials. Language, Cognition and Neuroscience, 29 (5):642-661, 2014.

Ellen F Lau, Colin Phillips, and David Poeppel. A cortical network for semantics:(de) constructing the N400. Nature Reviews Neuroscience, 9(12):920-933, 2008.

Steven J Luck. An introduction to the event-related potential technique. MIT press, 2014.

Jonathan Masci, Ueli Meier, Dan Cireşan, and Jürgen Schmidhuber. Stacked convolutional auto-encoders for hierarchical feature extraction. In International Conference on Artificial Neural Networks, pages 52-59. Springer, 2011.

Lee Osterhout and Phillip J Holcomb. Event-related brain potentials elicited by syntactic anomaly. Journal of Memory and Language, 31(6):785-806, 1992.

Adam Paszke, Sam Gross, Soumith Chintala, Gregory Chanan, Edward Yang, Zachary DeVito, Zeming Lin, Alban Desmaison, Luca Antiga, and Adam Lerer. Automatic differentiation in pytorch. 2017.

Jeffrey Pennington, Richard Socher, and Christopher Manning. Glove: Global vectors for word representation. In Proceedings of the 2014 conference on empirical methods in natural language processing (EMNLP), pages 1532-1543, 2014.

Matthew Peters, Mark Neumann, Mohit Iyyer, Matt Gardner, Christopher Clark, Kenton Lee, and Luke Zettlemoyer. Deep contextualized word representations. In Proceedings of the 2018 Conference of the North American Chapter of the Association for Computational Linguistics: Human Language Technologies, Volume 1 (Long Papers), volume 1, pages 2227-2237, 2018. 
Vitória Piai, Kristoffer Dahlslätt, and Eric Maris. Statistically comparing EEG/meg waveforms through successive significant univariate tests: How bad can it be? Psychophysiology, 52(3):440-443, 2015.

John Polich. On the relationship between eeg and p300: individual differences, aging, and ultradian rhythms. International journal of psychophysiology, 26 (1-3):299-317, 1997.

Milena Rabovsky, Steven S Hansen, and James L McClelland. Modelling the N400 brain potential as change in a probabilistic representation of meaning. Nature Human Behaviour, 2(9):693-705, 2018.

Alec Radford, Jeffrey Wu, Rewon Child, David Luan, Dario Amodei, and Ilya Sutskever. Language models are unsupervised multitask learners. Technical Report OpenAI, 2019.

Waseem Rawat and Zenghui Wang. Deep convolutional neural networks for image classification: A comprehensive review. Neural computation, 29(9):2352-2449, 2017.

Roland Schäfer. Processing and querying large web corpora with the cow14 architecture. In Proceedings of the 3rd Workshop on Challenges in the Management of Large Corpora (CMLC-3), page 2834, 2015.

Sara C Sereno, Keith Rayner, and Michael I Posner. Establishing a time-line of word recognition: evidence from eye movements and event-related potentials. Neuroreport, 9(10):2195-2200, 1998.

Nathaniel J Smith and Marta Kutas. Regression-based estimation of ERP waveforms: Ii. nonlinear effects, overlap correction, and practical considerations. Psychophysiology, 52(2):169-181, 2015.

Cyma Van Petten and Barbara J Luka. Prediction during language comprehension: Benefits, costs, and ERP components. International Journal of Psychophysiology, 83(2):176-190, 2012.

Shaorong Yan and T. Florian Jaeger. (Early) context effects on event-related potentials over natural inputs. Language, Cognition and Neuroscience, pages 1-22, 2019. 